\title{
Reconstruction of large-size abdominal wall defect using biodegradable poly-p-dioxanone mesh: an experimental canine study
}

\author{
Kenan Huang ${ }^{1 \dagger}$, Xinyu Ding ${ }^{1 \dagger}$, Benbo Lv ${ }^{1}$, Linyun Wei ${ }^{1}$, Juxian Sun ${ }^{1}$, Zhifei $X^{1}{ }^{1}$, Xiong Qin ${ }^{2^{*}}$ and Hua Tang ${ }^{1 *}$
}

\begin{abstract}
Background: Reconstruction of large-size abdominal wall defect (AWDs) is a huge challenge faced in current surgical practice. In this study, we aimed to evaluate the effectiveness and safety of biodegradable poly-p-dioxanone (PDO) mesh for reconstructing large-size AWDs in an experimental canine model.

Methods: Eighteen experimental canines were randomly and equally divided into three groups, namely, a PDO group, a Marlex group and a control group ( $n=6$ each). Following the creation of a $6 \mathrm{~cm} \times 5.5 \mathrm{~cm}$ AWD, PDO mesh and Marlex mesh were used to reconstruct the defect in the PDO and Marlex groups, respectively. The defect was closed using relaxation sutures alone in the control group. Animals were killed 24 weeks after surgery, and reconstruction outcomes were evaluated using radiography, histology and biomechanical testing.

Results: All animals except those in the control group survived the experiment. The PDO group showed no wound dehiscence, herniation or infection, whereas the animals in the Marlex group exhibited marked foreign body reactions. The PDO group had less intraabdominal adhesion than the Marlex group. As shown by radiography, histology and biomechanical testing, PDO mesh exhibited complete degradation and favorable biochemical strength at 24 weeks postsurgery.
\end{abstract}

Conclusions: PDO mesh implantation is an effective, safe treatment modality for reconstructing large-size AWDs.

Keywords: Abdominal wall defect, Canine model, Large size, Marlex, Mesh, Poly-p-dioxanone, Reconstruction

\section{Background}

Abdominal wall defects (AWDs) occur mainly after abdominal wall trauma or tumor resection and occasionally as congenital malformations. Small-size AWDs can be closed easily using the residual abdominal wall soft tissues; however, reconstruction of large-size AWDs normally require the use of prostheses and remain a huge challenge in current general surgical practice. The challenges are the hernias with rings greater than 10 $\mathrm{cm}$ in diameter, which are more likely to recur [1-4].

It is essential to optimize the choice of mesh materials for successful AWD reconstruction. A series of synthetic,

\footnotetext{
*Correspondence: Qin-Xiong@163.com; lordth1982@163.com

${ }^{\dagger}$ Equal contributors

${ }^{2}$ Department of Thoracic and Cardiovascular Surgery, First People's Hospital of Shanghai, Shanghai Jiaotong University, Shanghai, China

'Department of Thoracic and Cardiovascular Surgery, Changzheng Hospital, the Second Military Medical University, 415 Fengyang Road, Shanghai
} 200003, China

\section{Biomed Central

(c) 2014 Huang et al.; licensee BioMed Central Ltd. This is an Open Access article distributed under the terms of the Creative Commons Attribution License (http://creativecommons.org/licenses/by/2.0), which permits unrestricted use, distribution, and reproduction in any medium, provided the original work is properly credited. nondegradable mesh prostheses, such as Marlex [5] and polypropylene, have been examined in preclinical and clinical studies. However, use of polypropylene for reconstructing large-size AWDs can lead to some complications, such as chronic pain, abdominal wall stiffness, mesh dislocation and wound fistulas [6,7]. This material is also reported to be associated with a high risk of intraabdominal adhesion, which may lead to ileal and enteric fistulas [8-10]. Developments in materials science and technology have led to the evolution of mesh prostheses into biodegradable material, such as human acellular dermal matrix [11] and small-intestine submucosa [12]. These two materials are derived from human or animal tissue and are beneficial for tissue regeneration $[11,12]$, but they are primarily disadvantageous with regard to fast reabsorption and poor long-term mechanical strength. Furthermore, the cost of these biological meshes is 10 to 70 times greater than the cost of synthetic meshes, 
and some researchers have reported mixed results with regard to efficacy [13-16]. Composite meshes with protease-treated bovine skin collagen (atelocollagen) are currently available, but increased infection susceptibility and long-term coating failure have been reported $[17,18]$. Rapid degradation of biodegradable materials compromises the mechanical stability and consequently limits the use of these materials in large-size AWD reconstruction.

Poly-p-dioxanone (PDO) is a colorless, crystalline, resorbable polymer that is degraded by hydrolysis and completely metabolized in the body. It is available in different thicknesses $(0.15 \mathrm{~mm}$ perforated, $0.25 \mathrm{~mm}$ unperforated and $0.55 \mathrm{~mm}$ unperforated), is flexible but can preserve its shape, and can be fixed to cartilage with sutures. Thinner plates are resorbed within 25 weeks, and thicker plates are resorbed within 8 months $[19,20]$. The PDO mesh has been used for many years in chest wall reconstruction and orbital floor reconstruction. Researchers have demonstrated that it offers many advantages, such as excellent flexibility and elasticity, as well as suitable biocompatibility, which induces a minimal inflammatory response [21-24].

In our previous study, we attempted to use PDO mesh for chest wall defect reconstruction [25]. Our results showed that PDO mesh was superior to conventional bioabsorbable polymers mainly in terms of flexibility and elasticity. Moreover, this material also exhibited an appropriate reabsorption rate matching that of soft-tissue regeneration. A knowledge gap exists regarding the use of PDO mesh in large-size AWD reconstruction; therefore, we conducted an experimental canine study to evaluate the effectiveness and safety of PDO mesh alone in reconstructing large-size AWDs.

\section{Methods}

\section{Preparation of poly-p-dioxanone mesh}

Biodegradable PDO threads (Samyang, Seoul, South Korea) at a diameter of $0.8 \mathrm{~mm}$ were weaved into a mesh $7 \mathrm{~cm}$ in length and $6.5 \mathrm{~cm}$ in width at Donghua University, Shanghai, China. A scanning electron microscope was used for mesh ultrastructural analysis (Figure 1).

\section{Laboratory animals}

The study protocol was approved by the Animal Care and Use Committee of the Second Military Medical University, Shanghai, and carried out in accordance with the latest version of the US National Institutes of Health's Guide for the Care and Use of Laboratory Animals. Eighteen adult mongrel dogs of either sex, ages 1 to 2 years and weighing 15 to $18 \mathrm{~kg}$ were bred and housed at the Center for Laboratory Animals at the Second Military Medical University. Animals were randomly and equally divided into three groups according to the type of repair to be performed: a PDO group, a Marlex group (crystalline polypropylene and high-density polyethylene; Jia Te Plastics Co, Dongguan, China) and a control group ( $n=6$ for each group). The first two groups underwent large-size AWD reconstruction using PDO mesh or Marlex mesh, respectively and the control group underwent defect closure surgery without the use of any mesh prosthesis.

\section{Surgical procedure}

An intravenous injection of pentobarbital sodium (30 mg/kg; Shanghai Suolaibao Biotechnology, Shanghai, China) was given to induce general anesthesia, and an intravenous infusion of ketamine hydrochloride $(2 \mathrm{mg} /$ kg; Shanghai Suolaibao Biotechnology) and atracurium besylate $(0.3 \mathrm{mg} / \mathrm{kg}$; Shanghai Suolaibao Biotechnology) was given to maintain general anesthesia throughout the procedure. The animals were subsequently placed in the supine position while under general anesthesia with endotracheal intubation. The abdominal wall skin was shaved, sterilized with povidone-iodine (Shanghai Suolaibao Biotechnology) and draped as routinely done. A full-thickness midline xyphopubic AWD $(6 \mathrm{~cm} \times 5.5 \mathrm{~cm})$ was created, by which the fascia, underlying rectus abdominis muscle and peritoneum were resected (Figure 2A). In the PDO or Marlex mesh group, the mesh $(7 \mathrm{~cm} \times 6.5 \mathrm{~cm})$ was placed intraabdominally with a $0.5-\mathrm{cm}$ overlap and fixed tension-free to the abdominal wall with 2-0 polypropylene sutures (Ningbo Medical Needle Co, Ltd, Ningbo, China). Subsequently, the abdominal wall fascia was closed at the midline with a running 2-0 polypropylene suture, and the subcutis and skin were closed with interrupted 2-0 polypropylene sutures (Figures $2 \mathrm{~B}$ and $2 \mathrm{C}$ ). The defect was closed using relaxation sutures alone in the control group (Figure 2D). The endotracheal tube was removed when the animal resumed spontaneous breathing. After surgery, the animals were given an analgesic ( $1 \mathrm{ml}$ of buprenorphine) and an intramuscular injection of prophylactic 1,600,000-U procaine benzylpenicillin (Shanghai Suolaibao Biotechnology) and 80,000-U gentamicin sulfate (Shanghai Suolaibao Biotechnology) for the first three successive days. Animals were housed in single cages with a 12-hour day-night cycle, fed a commercially available diet and given free access to water. The animals were killed with an intravenous injection of 7,000 mg of pentobarbital sodium (Shanghai Suolaibao Biotechnology) and evaluated for clinical recurrence.

\section{Radiographic examination}

To further analyze the process of mesh degradation, abdominal computed tomography (CT) scans were taken at 12 and 24 weeks following AWD reconstruction while the animals were maintained under general anesthesia. Image processing and three-dimensional reconstruction were accomplished using the Advantage Workstation 4.2 (GE Healthcare, Shanghai, China). 

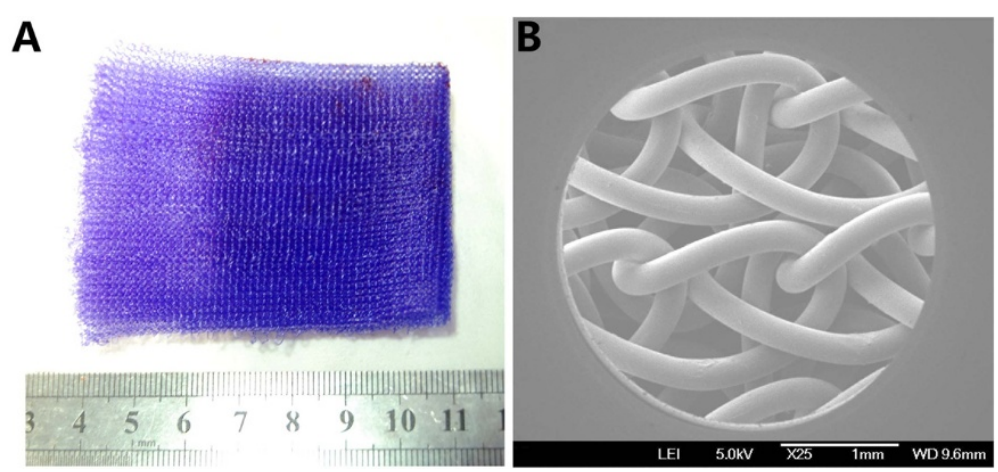

Figure 1 Poly-p-dioxanone mesh with a pore size of $\mathbf{0 . 2} \mathbf{~ m m}$. (A) Gross appearance. (B) Scanning electron microscopy image (original magnification, $\times 25$ ).

\section{Gross evaluation of wound adhesion}

The animals were killed at 24 weeks following AWD reconstruction. Gross wound adhesion was evaluated using a validated semiquantitative visual analogue scale ranging from a minimum of 0 to a maximum of 3 points $[26,27]$, with 0 signifying no significant adhesion; 1 indicating a thin, narrow, easily detachable adhesion; 2 meaning a thick adhesion limited to a single area; and 3 meaning a thick, broad adhesion involving the anterior or posterior abdominal wall and the viscera.

\section{Biomechanical test}

Freshly harvested regenerated soft-tissue samples were loaded onto a tensile testing machine (School of Materials
Science and Engineering Lab, Jiao Tong University, Shanghai, China) for tensile strength measurement with the speed calibrated at $100 \mathrm{~mm} / \mathrm{min}$ at $20^{\circ} \mathrm{C}$. The load rate was set at $0.5 \mathrm{~N} / \mathrm{mm}$, and the primary load was $1.5 \mathrm{~N}$. A stress-strain curve was plotted and fitted to produce the tensile strength and elastic modulus.

\section{Histological examination}

Regenerated soft-tissue samples were fixed in 10\% formaldehyde (Mengzhuang Bio-Technology Co, Ltd, Beijing, China) for 72 hours and decalcified in 15\% formic acid (Mengzhuang Bio-Technology Co, Ltd) for 2 to 6 weeks. Tissue samples were embedded in paraffin and cut into 5 - $\mu$ m-thick sections with a microtome for hematoxylin
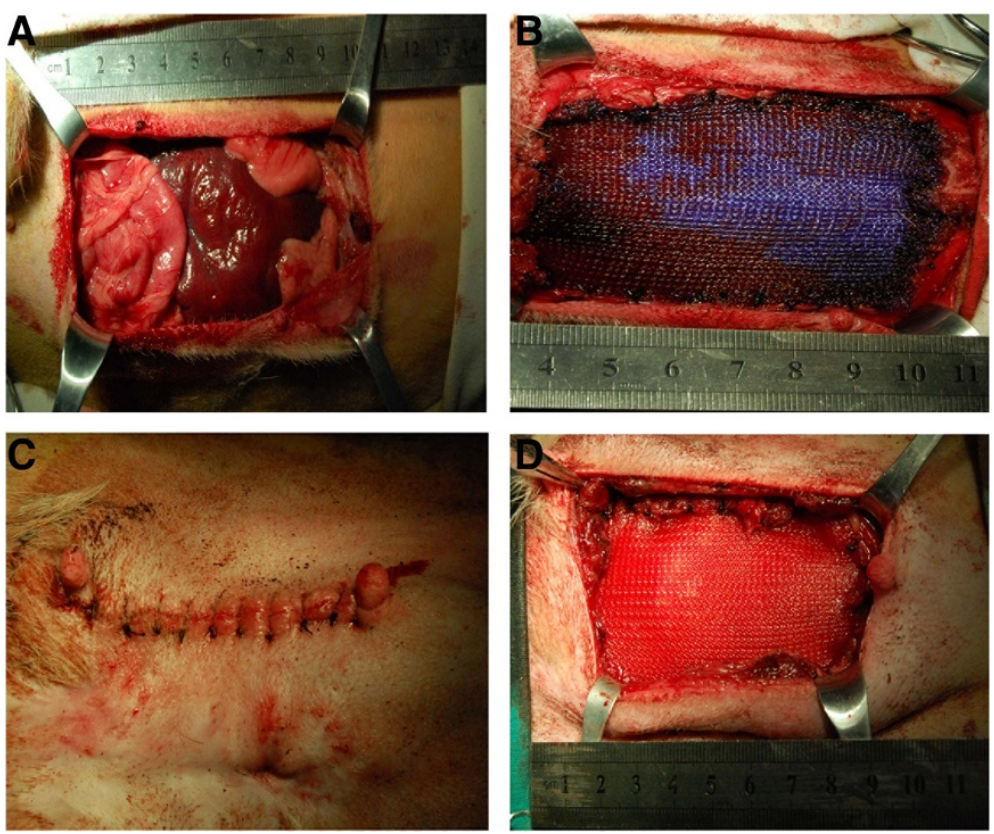

Figure 2 Surgical procedure performed for large-size abdominal wall defect reconstruction. A $6 \mathrm{~cm} \times 5.5 \mathrm{~cm}$ abdominal wall defect (AWD) (A) was created by removing bilateral rectus abdominis muscles. The AWD was reconstructed using poly-p-dioxanone mesh (B) or Marlex mesh (C). (D) The defect was closed using relaxation sutures in the control group. 
and eosin staining according to routine procedure. Native abdominal wall soft tissue was examined using the same protocol used for the controls.

\section{Statistical analysis}

The SPSS version 16.0 software (IBM SPSS, Chicago, IL, USA) was used for statistical analysis. All data are expressed as mean $\pm \mathrm{SD}$. The means were compared using one-way analysis of variance, and the two independent samples were analyzed by Student's $t$-test. $P<0.05$ was considered statistically significant.

\section{Results}

In the control group, all animals died immediately after surgery as a result of herniation. The animals in the other two groups survived the surgeries. The surviving animals exhibited good general well-being and normal activities at 4, 12 and 24 weeks postsurgery. All the surviving animals exhibited no wound dehiscence, herniation or infection (Figure 3A), except for one dog in the Marlex group that had a marked wound foreign body reaction and dehiscence (Figure 3B).

On abdominal CT scans, the radiopacity of the PDO mesh decreased but could still be observed at 12 weeks (Figure 4A). In contrast, Marlex mesh remained radiopaque throughout the 12 weeks after surgery (Figure 4B). This radiopacity had mostly disappeared at 24 weeks, indicating that the PDO mesh had nearly completely degraded (Figure 4C). Furthermore, we observed obvious shrinkage in the Marlex mesh group, but not in the PDO mesh group (Figure 4D).

The proportion of adhesion was recorded for each group. Statistical analysis of the adhesion scores was then performed. The mean adhesion score of the PDO mesh group was $1.1 \pm 0.2$, and the mean Marlex mesh group score was $2.6 \pm 0.2$. Overall, PDO mesh had a significantly lower gross wound adhesion score than Marlex mesh $(P<0.05)$ (Figure 5A). All PDO meshes became completely degraded at 24 weeks postsurgery with easily detachable adhesions to the peritoneum and the omentum (Figure 5B). Marlex mesh showed no marked degradation, but had extensive dense adhesion to the omentum and the visceral organs, including the omentum and the colon (Figure 5C).

To compare the normal abdominal wall and evaluate the mechanical properties of the reconstructed abdominal wall, the tensile strength of the implanted biomaterials was measured at 24 weeks after surgery. The Marlex group had significantly greater tensile strength than the PDO group and the control group (PDO vs. Marlex vs. control, $22.8 \pm 0.4 \mathrm{~N}$ vs. $18.2 \pm 0.3 \mathrm{~N}$ vs. $18.3 \pm 0.3 \mathrm{~N}$; $P=0.000$ ), whereas the PDO group exhibited tensile strength similar to that of the control group $(P=0.664)$ (Figures 6A and 6B).

PDO mesh exhibited almost complete degradation on histological examination at 24 weeks postsurgery, and a small amount of mesh residuals were enveloped by the regenerated soft tissues and surrounded by extensive fibroconnective tissues (Figure 7A). Marlex mesh showed no microscopic degradation either (Figure 7B).

\section{Discussion}

An optimal mesh prosthesis for reconstructing AWDs should be easily fashioned but resistant to moisture, disinfection or mechanical tension; should not be immunogenic or carcinogenic; and should not cause chemical, inflammatory, foreign body, allergic or hypersensitivity reactions [26]. None of the currently available prosthesis materials meet all the aforementioned requirements, although nondegradable materials remain the mainstay choice for large-size AWD reconstruction. However, use of these nonbiological, nondegradable prostheses, such as alloy fabrics and high-molecular-mass polymers, carries a high risk of long-term complications.

Biological materials derived from autologous tissues (fascia, muscle flap and autogenous dermis) [28], allogeneic tissue (amniotic membrane) [29] and xenogeneic tissues (porcine heart valve and bovine peritoneum)

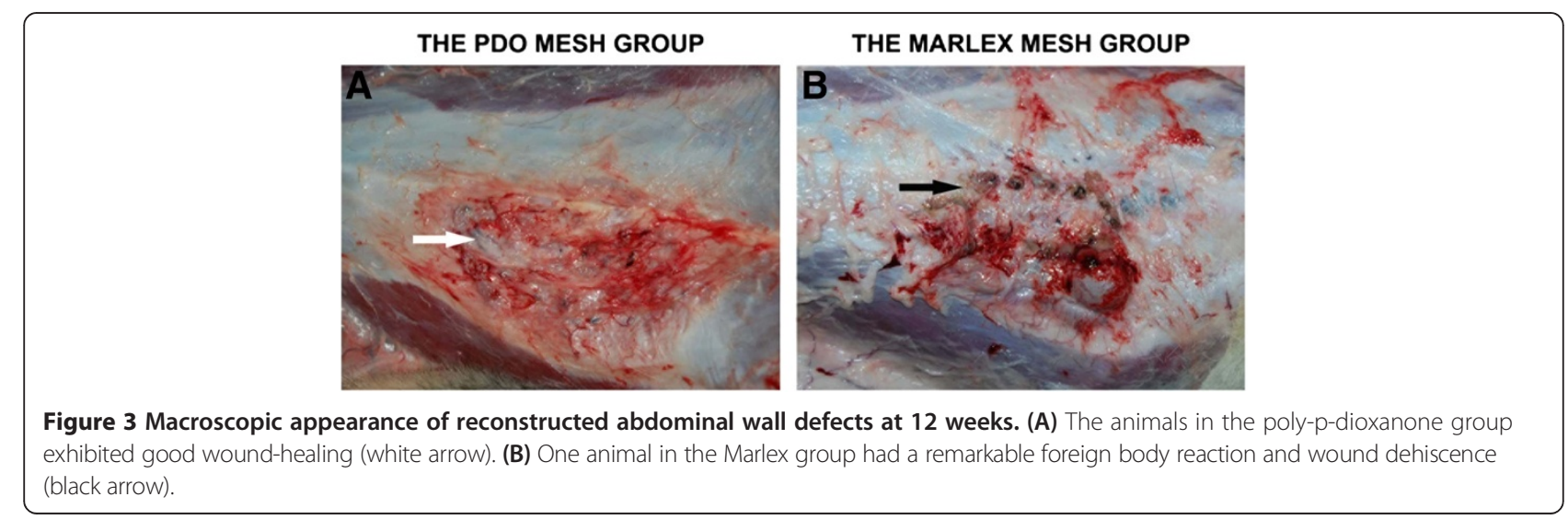




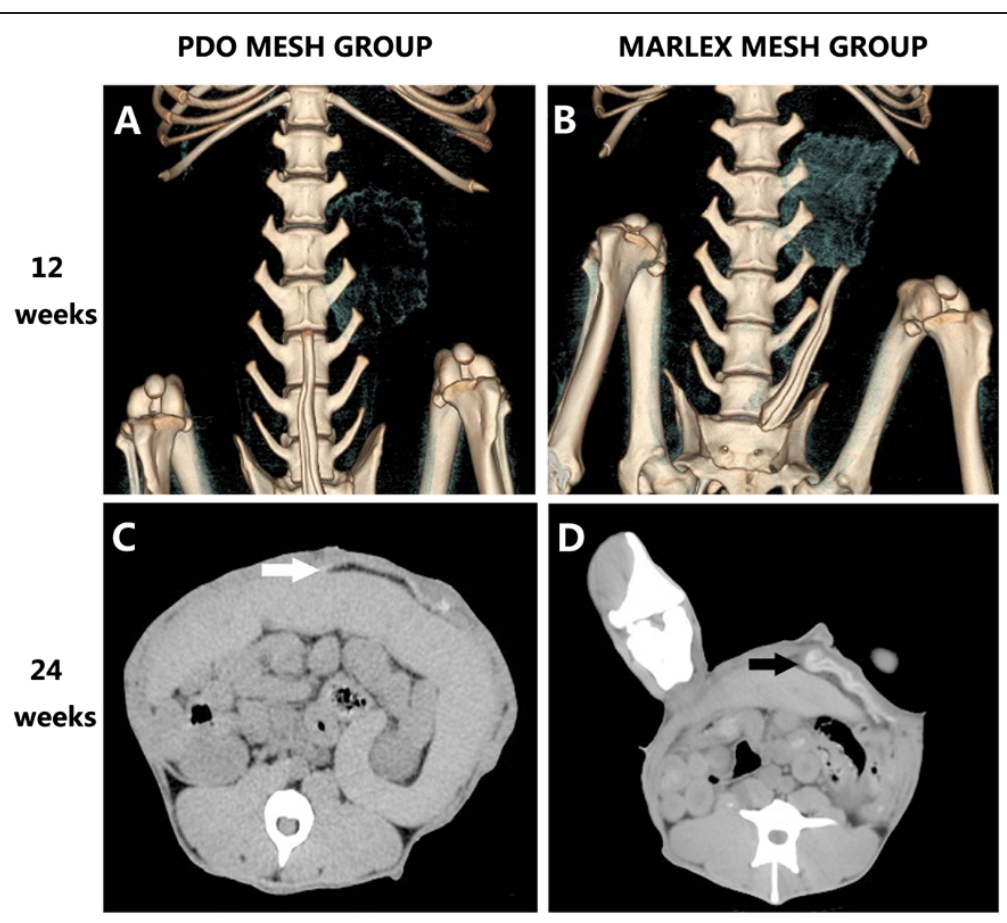

Figure 4 Three-dimensional reconstructions of abdominal computed tomography scans. At 12 weeks, The poly-p-dioxanone (PDO) mesh (A) and the Marlex mesh (B). At 24 weeks, On abdominal CT scans, the poly-p-dioxanone (PDO) mesh (black arrow in (C)) and the Marlex mesh (white arrow in (D)).

A
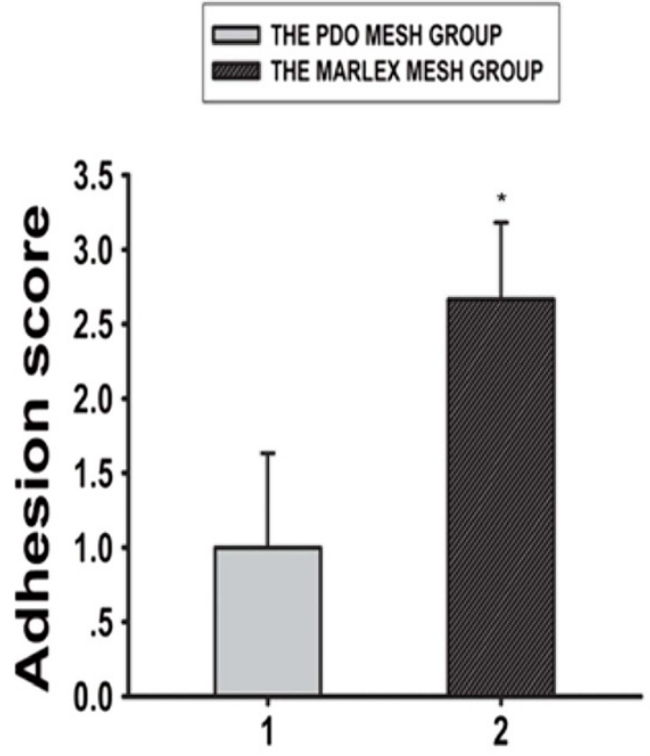
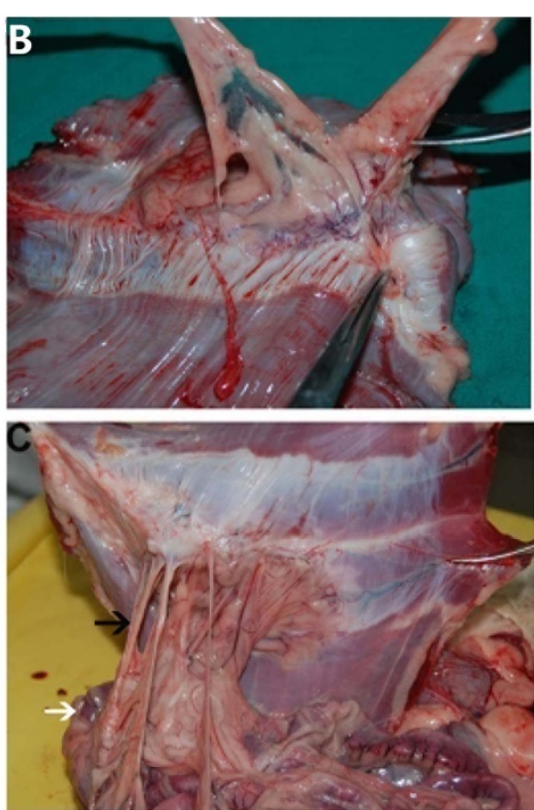

Figure $\mathbf{5}$ Macroscopic evaluation of wound adhesion of reconstructed abdominal wall at $\mathbf{2 4}$ weeks. (A) Overall, poly-p-dioxanone (PDO) mesh had a significantly lower gross wound adhesion score than Marlex mesh $\left({ }^{*} P<0.05\right)$. (B) All PDO meshes became completely degraded at 24 weeks postsurgery with easily detachable adhesions to the peritoneum and the omentum. (C) Marlex mesh showed extensive dense adhesions to the omentum and the visceral organs, including the omentum and the colon. The black and white arrows indicate the omentum and the colon, respectively. 

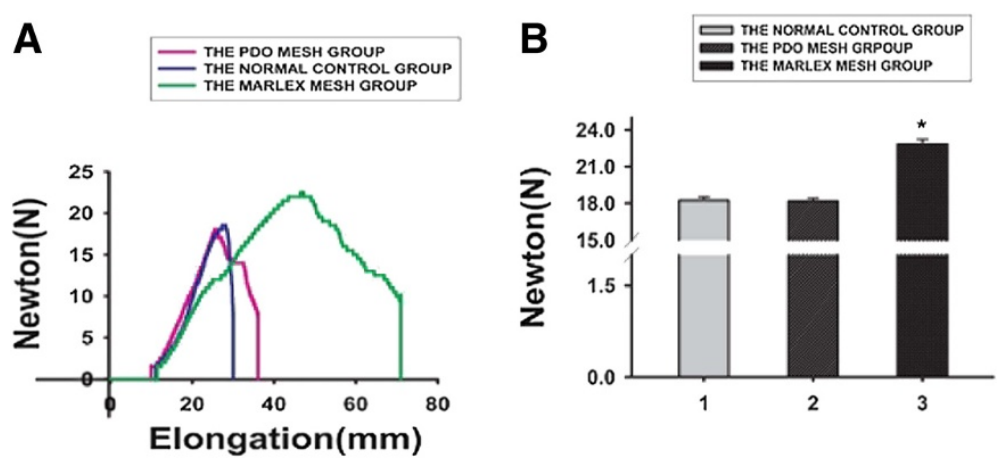

Figure 6 Mechanical properties of implanted biomaterials at 24 weeks after surgery. (A) Ultimate tensile strength of each group. (B) The statistical analysis of the ultimate tensile strength. Data are mean $\pm \operatorname{SD}(n=6) . P<0.05$. PDO, Poly-p-dioxanone.

[30] are beneficial for tissue regeneration in the recipient site with a relatively lower long-term risk than nonbiological materials. However, AWD reconstruction using allograft is associated with a high risk of herniation recurrence, mainly due to reduction in biological mesh tensile strength and increase in elastin content in human dermis [29]. Moreover, biomechanical properties of biological meshes are highly variable between donors [31].

Artificial synthetic mesh was first introduced by Usher in 1958 [5], and it is widely used in general surgical practice because of its favorable biocompatibility and mechanical stability. As a nondegradable material, prosthetic mesh, such as Marlex mesh, is associated with a series of postoperative adverse effects, including persistent pain, hematoma, wound erosion, infection and herniation, and even enterocutaneous fistula [1,32,33]. McKenna et al. [34] reported an approximately $25 \%$ infection rate associated with Marlex mesh implantation. Serious cases normally require a second-look surgery to remove the infectious prosthesis [34]. A large number of newly emerging tissue engineering materials have been developed for reconstructing human body wall defects in preclinical and clinical studies based on breakthroughs in materials science and technology [35-38]. PDO is a synthetic absorbable polymer that has been used for surgical suture weaving. In our previous experimental canine model study, we demonstrated that chest wall defects can be structurally and functionally reconstructed by using a composite implant containing PDO mesh [37]. In the present study, we also successfully reconstructed largesize AWDs by using PDO mesh alone. To the best of our knowledge, this study is the first to report the use
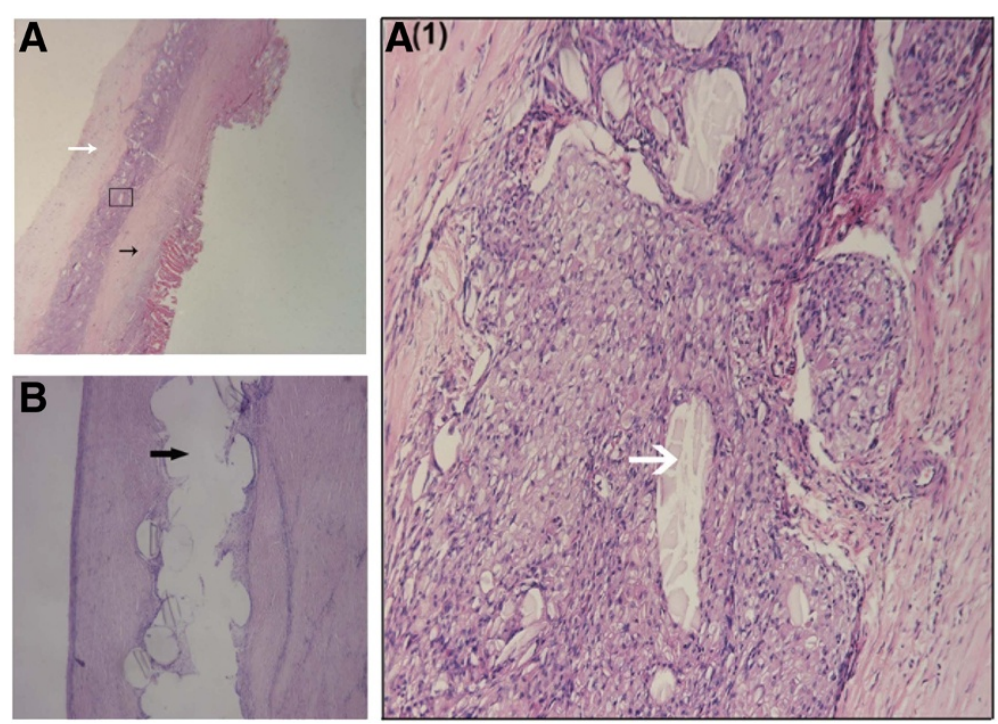

Figure 7 Histological images of the poly-p-dioxanone and Marlex meshes. (A) The white and black arrows indicate the inner and outer sides of the reconstructed abdominal wall, respectively. (A(1)) Detailed, high-magnification image of the boxed area in (A) (original magnification, $\times 100)$. The white arrow points to the residual poly-p-dioxanone (PDO) mesh. (B) The black arrow indicates the absence of soft-tissue regeneration in the Marlex mesh. Images in (A) and (B) were stained with hematoxylin and eosin (original magnification, $\times 10$ ). 
of synthetic absorbable polymer alone for reconstructing experimental large-size AWDs.

In our study, PDO mesh showed good biocompatibility. No animals in the PDO group had wound dehiscence, herniation or infection, whereas one animal exhibited remarkable foreign body reaction and consequent wound nonhealing in the Marlex group. Furthermore, histological evidence showed that PDO mesh expedited soft-tissue regeneration by inducing collagen deposition and neoangiogenesis with a reasonable degradation rate over the course of 24 weeks. In contrast, Marlex mesh showed no signs of absorption or degradation within this period. Researchers in similar previous studies [13] have found that microscopically intact polypropylene mesh filaments were surrounded by variably organized fibrous tissue, and, as expected, a pronounced foreign body reaction and intraabdominal adhesion formation.

Tissue adhesion usually occurs following fibrin exudation in the wound. Our results show that PDO mesh implantation caused only mild wound adhesion compared to Marlex mesh. As a degradable material, PDO mesh is expected to elicit minimal foreign body reaction and inflammatory response. Shrinkage in mesh implants is a major adverse effect, regardless of the use of degradable or nondegradable material. This reduction results mainly from chronic scarring and scar retraction and consequent distortion or dislocation of the implanted mesh. The presence of an irregularly shaped mesh surface will induce foreign body and inflammatory reactions, which are destructive of the newly regenerated tissue and makes tissue prone to infection and the formation of fistula. Our follow-up CT scans showed that remarkable mesh shrinkage occurred in the Marlex group, but not in the PDO group. Previous histological studies have also confirmed that PDO mesh implantation was associated with less serious inflammation and fibrosis, formation of smaller granulomas and less cell turnover and remodeling [39]. More importantly, once the initial infection has developed, only antibiotic gauze needs to be applied to the wound during dressing change. For synthetic, nondegradable mesh prostheses, however, the mesh has to be taken off. When bacteria enter the surgical incision, the development of a mesh infection is dependent on the bacteria adherent to the prosthetic material. Only a clean wound can heal rapidly and without infection.

A major technical concern regarding biodegradable mesh prostheses is long-term biomechanical strength. No wound herniation occurred in the PDO group over the course of 24 weeks in our present study. Postoperative 4-week follow-up CT scans showed that PDO mesh, just as nondegradable Marlex mesh, maintained a wellpreserved shape and provided adequate mechanical support for AWD reconstruction at an early phase. Furthermore, 12- and 24-week postoperative CT scans suggested that PDO mesh-regenerated tissue had sufficient tensile and burst strength to withstand abdominal wall tension, although the mesh had become degraded. Biomechanical testing results also confirmed that PDO meshregenerated tissue had favorable mechanical strength similar to the native abdominal wall in the midterm sense, although it was even higher in Marlex mesh.

This study has some limitations. First, for the protection of the animals, this study had a relatively small sample size, with only six animals in each group. Second, the follow-up period was only 24 weeks, and therefore it remains unknown whether PDO mesh implantation was effective and safe for large-size AWD reconstruction. In addition, the PDO mesh was not found to be biodegraded completely at 24 weeks postsurgery, hence it is still likely to have retained sufficient tensile strength to prevent failure at the surgical site and subsequent herniation. Therefore, long-term observation is necessary in future research. Third, PDO mesh could still induce some intraabdominal adhesions. The most probable cause is the fact that our PDO mesh was not coated with any natural macromolecular biomaterial. It is known that additional coating with artificial or biological polymers, such as collagen, can effectively prevent the formation of postoperative wound adhesions [40-42].

\section{Conclusions}

PDO mesh is a good alternative to Marlex mesh for largesize AWD reconstruction. PDO mesh has a reasonable degradation rate, good biocompatibility and sufficient biomechanical strength similar to the native abdominal wall in rectus abdominis. The effectiveness and safety of using PDO mesh in reconstructing large-size AWD needs to be validated in further long-term preclinical and clinical studies.

\section{Competing interests}

The authors declare that they have no competing interests.

\section{Authors' contributions}

HT participated in the design of the study. BLv and LW performed the statistical analysis. JS interpreted the data. XD participated in its design and coordination and helped to draft the manuscript. KH participated in the sequence alignment and drafted the manuscript. XQ critically revised the manuscript for important intellectual content. All authors read and approved the final manuscript.

\section{Acknowledgements}

The authors would like to thank Jim Kretlow, Wei Liu, Guangpeng Liu, Yilin Cao and Guangdong Zhou, Shanghai Key Laboratory of Tissue Engineering, Shanghai Ninth People's Hospital.

Received: 4 March 2013 Accepted: 19 February 2014

Published: 14 March 2014

\section{References}

1. Leber GE, Garb JL, Alexander Al, Reed WP: Long-term complications associated with prosthetic repair of incisional hernias. Arch Surg 1998, 133:378-382.

2. Sukkar SM, Dumanian GA, Szczerba SM, Tellez MG: Challenging abdominal wall defects. Am J Surg 2001, 181:115-121. 
3. Wong $\mathrm{CH}$, Lin $\mathrm{CH}$, Fu B, Fang JF: Reconstruction of complex abdominal wall defects with free flaps: indications and clinical outcome. Plast Reconstr Surg 2009, 124:500-509.

4. Rohrich RJ, Lowe JB, Hackney FL, Bowman JL, Hobar PC: An algorithm for abdominal wall reconstruction. Plast Reconstr Surg 2000, 105:202-217.

5. Usher FC, Ochsner J, Tuttle LL Jr: Use of Marlex mesh in the repair of incisional hernias. Am Surg 1958, 24:969-974.

6. Kingsnorth A, LeBlanc K: Hernias: inguinal and incisional. Lancet 2003 362:1561-1571.

7. Binnebösel $M$, von Trotha $K T$, Jansen $P L$, Conze J, Neumann UP, Junge $K$ Biocompatibility of prosthetic meshes in abdominal surgery. Semin Immunopathol 2011, 33:235-243.

8. Burger JW, Luijendijk RW, Hop WC, Halm JA, Verdaasdonk EG, Jeekel J: Long-term follow-up of a randomized controlled trial of suture versus mesh repair of incisional hernia. Ann Surg 2004, 240:578-585.

9. Bringman $S$, Blomqvist $P$ : Intestinal obstruction after inguinal and femoral hernia repair: a study of 33,275 operations during 1992-2000 in Sweden. Hernia 2005, 9:178-183.

10. Matthews BD, Pratt BL, Pollinger HS, Backus CL, Kercher KW, Sing RF, Heniford BT: Assessment of adhesion formation to intra-abdominal polypropylene mesh and polytetrafluoroethylene mesh. J Surg Res 2003, 114:126-132.

11. Baillie DR, Stawicki SP, Eustance N, Warsaw D, Desai D: Use of human and porcine dermal-derived bioprostheses in complex abdominal wall reconstructions: a literature review and case report. Ostomy Wound Manage 2007, 53:30-37.

12. Holton LH 3rd, Kim D, Silverman RP, Rodriguez ED, Singh N, Goldberg NH: Human acellular dermal matrix for repair of abdominal wall defects: review of clinical experience and experimental data. J Long Term Eff Med Implants 2005, 15:547-558

13. Peeters E, van Barneveld KWY, Schreinemacher MH, De Hertogh G, Ozog $Y$, Bouvy N, Miserez M: One-year outcome of biological and synthetic bioabsorbable meshes for augmentation of large abdominal wall defects in a rabbit model. J Surg Res 2013, 180:274-283.

14. Blatnik J, Jin J, Rosen M: Abdominal hernia repair with bridging acellular dermal matrix: an expensive hernia sac. Am J Surg 2008, 196:47-50.

15. Schuster $R$, Singh J, Safadi BY, Wren SM: The use of acellular dermal matrix for contaminated abdominal wall defects: wound status predicts success. Am J Surg 2006, 192:594-597.

16. Catena F, Ansaloni L, Gazzotti F, Gagliardi S, Di Saverio S, D'Alessandro L, Pinna AD: Use of porcine dermal collagen graft (Permacol) for hernia repair in contaminated fields. Hernia 2007, 11:57-60.

17. van't Riet M, van Steenwijk PJ d V, Bonjer HJ, Steyerberg EW, Jeekel J: Mesh repair for postoperative wound dehiscence in the presence of infection: is absorbable mesh safer than non-absorbable mesh? Hernia 2007, 11:409-413.

18. Schreinemacher MH, Emans PJ, Gijbels MJ, Greve JW, Beets GL, Bouvy ND: Degradation of mesh coatings and intraperitoneal adhesion formation in an experimental model. Br J Surg 2009, 96:305-313.

19. Rimmer J, Ferguson LM, Saleh HA: Versatile applications of the polydioxanone plate in rhinoplasty and septal surgery. Arch Facial Plast Surg 2012, 14:323-330.

20. Boenisch $M$, Mink A: Clinical and histological results of septoplasty with a resorbable implant. Arch Otolaryngol Head Neck Surg 2000, 126:1373-1377.

21. Boenisch M, Tamás H, Nolst Trenité GJ: Influence of polydioxanone foil on growing septal cartilage after surgery in an animal model: new aspects of cartilage healing and regeneration (preliminary results). Arch Facial Plast Surg 2003, 5:316-319.

22. Puma F, Ragusa M, Daddi G: Chest wall stabilization with synthetic reabsorbable material. Ann Thorac Surg 1992, 53:408-411.

23. Mäkelä $P$, Pohjonen T, Törmälä $P$, Waris T, Ashammakhi N: Strength retention properties of self-reinforced poly L-lactide (SR-PLLA) sutures compared with polyglyconate (Maxon) and polydioxanone (PDS) sutures: an in vitro study. Biomaterials 2002, 23:2587-2592

24. Kontio R, Ruuttila P, Lindroos L, Suuronen R, Salo A, Lindqvist C, Virtanen I, Konttinen YT: Biodegradable polydioxanone and poly $(L / D)$ lactide implants: an experimental study on peri-implant tissue response. Int Oral Maxillofac Surg 2005, 34:766-776.

25. Qin X, Tang $H$, Xu Z, Zhao X, Sun Y, Gong Z, Duan L: Chest wall reconstruction with two types of biodegradable polymer prostheses in dogs. Eur J Cardiothorac Surg 2008, 34:870-874.
26. Silverman RP, Li EN, Holton LH 3rd, Sawan KT, Goldberg NH: Ventral hernia repair using allogeneic acellular dermal matrix in a swine model. Hernia 2004, 8:336-342

27. Ko JH, Salvay DM, Paul BC, Wang EC, Dumanian GA: Soft polypropylene mesh, but not cadaveric dermis, significantly improves outcomes in midline hernia repairs using the components separation technique. Plast Reconstr Surg 2009, 124:836-847

28. Ionescu S, Andrei B, Tirlea S, Bunea B, Licsandru E, Cirstoveanu C, Bizubac M, Ivanov M, Shelleh M, Gurita A, Tabacaru R: Considerations on gastroschisis repair. Chirurgia (Bucur) 2013, 108:509-515

29. Avison DL, DeFaria W, Tryphonopoulos P, Tekin A, Attia GR, Takahashi H, Jin Y, Palaios E, Pararas N, Carreno MR, Santiago S, Bazer F, Ruiz P, Tzakis A: Heterotopic uterus transplantation in a swine model. Transplantation 2009, 88:465-469.

30. Clarke KM, Lantz GC, Salisbury SK, Badylak SF, Hiles MC, Voytik SL: Intestine submucosa and polypropylene mesh for abdominal wall repair in dogs. J Surg Res 1996, 60:107-114.

31. Gobin AS, Butler CE, Mathur AB: Repair and regeneration of the abdominal wall musculofascial defect using silk fibroin-chitosan blend. Tissue Eng 2006, 12:3383-3394.

32. Chand M, On J, Bevan K, Mostafid H, Venkatsubramaniam AK: Mesh erosion following laparoscopic incisional hernia repair. Hernia 2012, 16:223-226.

33. Robinson TN, Clarke JH, Schoen J, Walsh MD: Major mesh-related complications following hernia repair: events reported to the food and drug administration. Surg Endosc 2005, 19:1556-1560.

34. McKenna RJ Jr, Mountain CF, McMurtrey MJ, Larson D, Stiles QR: Current techniques for chest wall reconstruction: expanded possibilities for treatment. Ann Thorac Surg 1988, 46:508-512.

35. Tang H, Xu Z, Qin X, Wu B, Wu L, Zhao X, Li Y: Chest wall reconstruction in a canine model using polydioxanone mesh, demineralized bone matrix and bone marrow stromal cells. Biomaterials 2009, 30:3224-3233.

36. Peeters $G$, Decloedt J, Nagels $H$, Cambier B: Treatment of the severe or recurrent inverted nipple by interposition of a resorbable polydioxanone sheet. J Plast Reconstr Aesthet Surg 2010, 63:e175-e176.

37. Kalfa D, Bel A, Chen-Tournoux A, Della Martina A, Rochereau P, Coz C, Bellamy V, Bensalah M, Vanneaux V, Lecourt S, Mousseaux E, Bruneval P, Larghero J, Menasché P: A polydioxanone electrospun valved patch to replace the right ventricular outflow tract in a growing lamb model. Biomaterials 2010, 31:4056-4063.

38. Deorio JK, Ware AW: Single absorbable polydioxanone pin fixation for distal chevron bunion osteotomies. Foot Ankle Int 2001, 22:832-835.

39. Otto J, Binnebösel M, Pietsch S, Anurov M, Titkova S, Ottinger AP, Jansen M, Rosch R, Kämmer D, Klinge U: Large-pore PDS mesh compared to small-pore PG mesh. J Invest Surg 2010, 23:190-196.

40. Vlabos A, Yu P, Lucas CE, Ledgerwood AM: Effect of a composite membrane of chitosan and poloxamer gel on postoperative adhesive interactions. Am Surg 2001, 67:15-21.

41. Costain DJ, Kennedy R, Ciona C, McAlister VC, Lee TD: Prevention of postsurgical adhesions with $\mathrm{N}, \mathrm{O}$-carboxymethyl chitosan: examination of the most efficacious preparation and the effect of $\mathrm{N}, \mathrm{O}$-carboxymethyl chitosan on postsurgical healing. Surgery 1997, 121:314-319.

42. van't Riet M, Burger JW, Bonthuis F, Jeekel J, Bonjer HJ: Prevention of adhesion formation to polypropylene mesh by collagen coating: a randomized controlled study in a rat model of ventral hernia repair. Surg Endosc 2004, 18:681-685.

doi:10.1186/1477-7819-12-57

Cite this article as: Huang et al:: Reconstruction of large-size abdominal wall defect using biodegradable poly-p-dioxanone mesh: an experimental canine study. World Journal of Surgical Oncology 2014 12:57. 